\title{
CONCENTRACIONES DE METALES PESADOS EN MURCIÉLAGOS DEL LODGE “COCK OF THE ROCKS” Y ALREDEDORES, KOSÑIPATA, CUZCO, PERÚ
}

\section{HEAVY METAL CONCENTRATIONS IN BATS AROUND AND INSIDE THE LODGE "COCK OF THE ROCKS", KOSÑIPATA, CUZCO, PERU}

\author{
Marta Williams ${ }^{1}$, Daniel Ramos ${ }^{2}$, Alfredo Butrón ${ }^{3}$, Sofía Gonzales-Zúñiga ${ }^{3}$, Natalia Ortiz ${ }^{3}$ y Braulio La Torre ${ }^{4}$
}

\begin{abstract}
Resumen
Durante las épocas seca (julio, 2007) y lluviosa (marzo y abril, 2008), se colectaron murciélagos de los géneros Carollia, Anoura y Sturnira dentro y en los alrededores del Lodge "Cock of the Rocks" (Kosñipata, Cuzco, Perú). Los hígados y páncreas de los individuos colectados fueron utilizados para medir las concentraciones de plomo, cadmio, cobre y zinc mediante un espectrofotómetro de absorción atómica. El objetivo del presente trabajo fue determinar las diferencias en los niveles de metales pesados en murciélagos de tres géneros y distintas especies, además de establecer variaciones entre los dos períodos estacionales. Se encontraron diferencias significativas $(\alpha=0,05)$ en las concentraciones de los metales entre los géneros, más no entre las épocas. La concentración en partes por millón (ppm) de plomo varió entre 0 y 15.15 , la de cadmio entre 0 y 19.05 , la de cobre entre 4.38 y 81.82 , y la de zinc entre 9.49 y 284. En general, los mayores valores de cobre y zinc se deben a que estos metales suplen necesidades fisiológicas en los mamíferos, por lo que son requeridos en mayores cantidades. Las concentraciones varían entre las especies, individuos e incluso en éstos a lo largo del tiempo, de acuerdo al tipo de dieta, grado de exposición y de asimilación. Aparentemente, este sería el primer informe de concentraciones de metales pesados en murciélagos de Sudamérica.
\end{abstract}

Palabras clave: murciélagos, Kosñipata, Cuzco, hígado, páncreas, plomo, cadmio, cobre, zinc, metales pesados.

\begin{abstract}
During the dry season (July 2007) and the wet season (March and April 2008), bats of the genera Carollia, Sturnira and Anoura were collected inside and around the Lodge "Cock of the Rocks" (Kosñipata, Cuzco, Peru). The liver and pancreas of all collected individuals were used to measure lead, cadmium, copper and zinc concentrations using an atomic absorption spectrophotometer. The interest was focused in determining differences in the levels of those metals among genera and species, as well as in establishing variations in metal concentrations during both periods. Significant differences $(\alpha=0.05)$ were found in metal concentrations among genera, but not among seasons. Parts per million ( $\mathrm{ppm}$ ) concentrations of lead ranged from 0 to 15.15 , cadmium varied from 0 to 19.05 , copper from 4.38 to 81.82 and zinc from 9.49 to 284 . In general, higher values of copper and zinc were found due to the fact that mammals may require them in larger quantities to meet their physiological needs. Metal concentrations vary among species, individuals and even in themselves over time, according to the type of diet, degree of exposure and assimilation rate. Apparently, this is a first report of heavy metal concentrations in bats from South America.
\end{abstract}

Key words: bats, Kosñipata, Cuzco, liver, pancreas, lead, cadmium, copper, zinc, heavy metals.

\section{Introducción.}

La concentración de metales se ha elevado en la naturaleza debido a la industrialización y al uso de productos que los contienen (Albert, 1997; Capó, 2002). Al ser emitidos a la atmósfera, los elementos metálicos pueden transportarse por el viento para luego depositarse en el suelo, agua, vegetación y fauna (McDowell, 1992; Albert, 1997). Los metales pesados corresponden a un grupo de elementos con características químicas semejantes (Ahumada, 1994; Gil et al., 2006) y al encontrarse en bajas concentraciones en los organismos, son denominados trazas. Sin embargo, debe tomarse en cuenta el hecho que la toxicidad de los metales en los organismos no se da por su naturaleza química sino por su concentración (Jacinto \& Aguilar, 2007). Es decir, no puede considerarse que existan elementos tóxicos per se, sino concentraciones tóxicas de dichos elementos (McDowell, 1992). 
Las causas principales de captación de metales pesados en los mamíferos, son la vía respiratoria y la ingestión durante la alimentación (Milton \& Johnson, 1999). Por otro lado, las diferencias en las concentraciones de metales pesados como plomo, cadmio, cobre y zinc, pueden darse debido al grado de exposición a la fuente contaminante, al tipo de dieta o a la variación en la asimilación existente entre las especies y hasta entre individuos (Hoenerhoff \& Williams, 2004; Walker et al., 2007). Inclusive, las concentraciones mínimas y de tolerancia varían de animal en animal y periódicamente en el mismo.

Tanto los insectos como las plantas, fuentes principales de alimento, pueden acumular metales y transferirlos a los murciélagos al ser ingeridos. Sin embargo, los individuos más jóvenes, preñados, lactantes, desnutridos o enfermos muestran mayor sensibilidad o requerimiento (Eckert \& Randall, 1990; McDowell, 1992; Walker et al., 2007). Además, la época puede influir en las concentraciones de metales, ya que varían la disponibilidad de alimento, la entrada de minerales al entorno y la absorción de los elementos por parte de las plantas (McDowell, 1992; Espinoza, 2000). En los animales, los metales son bioacumulados y excretados por órganos tales como el hígado y el páncreas (McDowell, 1992; Albert, 1997; Milton \& Johnson, 1999).

La importancia de conocer los niveles de los metales pesados en los quirópteros, radica en que éstos cumplen un papel crucial como agentes polinizadores y controladores de plagas, e incluso como biomonitores para determinar niveles de contaminación en un determinado ecosistema (Hoenerhoff \& Williams, 2004; Walker et al., 2007). Dada su diversidad (Patterson et al., 1996), a los quirópteros se les atribuye un alto nivel de interacción en los ecosistemas.

Los objetivos del estudio se centraron en establecer las diferencias en los niveles de cadmio, plomo, cobre y zinc, en hígados y páncreas de individuos de los géneros Carollia, Anoura y Sturnira. Además, se establecieron diferencias entre las épocas seca (de mayo a setiembre) y lluviosa (de octubre a abril). También se determinaron las concentraciones a nivel de especies en el Lodge "Cock of the Rocks" y sus alrededores.

\section{Materiales y métodos.}

Área de estudio.

El estudio se realizó en el Lodge "Cock of the Rocks" (71 $\left.32^{\prime} 47^{\prime \prime} \mathrm{O}, 13^{\circ} 03^{\prime} 20^{\prime \prime} \mathrm{S}\right)$ y sus alrededores, en el distrito de Kosñipata, provincia de Paucartambo, departamento de Cuzco, Perú, perteneciente a la zona de amortiguamiento del Parque Nacional Manu, entre los 1180 y 1640 msnm.

Esta zona, caracterizada por la presencia de bosque nublado presenta dos periodos estacionales: la época seca, con menores precipitaciones y temperaturas, correspondiendo a los meses de mayo a setiembre, y la época lluviosa con abundantes lluvias y altas temperaturas, extendiéndose de octubre a abril (INRENA \& Pro Manu, 2002). Los bosques del área de estudio pertenecen a la zona de vida bosque muy húmedo montano bajo subtropical (bmh-MBS), cuyo relieve es accidentado, con suelos delgados y de naturaleza acida. La vegetación es densa, alta $y$ siempre verde, predominando árboles de los géneros Podocarpus, Weinmennia, Moenas, Didimopanax, Clusia, Rapanea, Solanum, entre otras. En el sotobosque se encuentran helechos de los géneros Cyathea, Alsophila y Dicksonia, además de palmeras de los géneros Caroxylon y Geomona (Municipalidad Provincial de Paucartambo, 2006; Delgado ,2010). Metodología.

Se utilizaron redes de neblina de 6 a 12 metros de largo y 2.5 de ancho, las cuales fueron colocadas en zonas consideradas de tránsito, forrajeo y/o refugio, dentro del sotobosque. Se capturaron murciélagos de los géneros Carollia, Anoura, Sturnira y otros menos abundantes (Philoderma, Artibeus y Platyrrhinus), durante 17 días en Julio del 2007 (época seca) y 10 días entre marzo y abril del 2008 (época lluviosa). Se colectaron y disectaron 54 individuos, conservándose sus hígados y páncreas en alcohol de $70^{\circ}$.

Se reunió el hígado y el páncreas en una sola muestra por individuo, analizándose las concentraciones de plomo $(\mathrm{Pb})$, cadmio $(\mathrm{Cd})$, cobre $(\mathrm{Cu})$ y zinc $(\mathrm{Zn})$ con un espectrofotómetro de absorción atómica (marca Perkin Elmer, modelo Analysis 200) con un nivel de detección de $0.01 \mathrm{ppm}$. Los análisis se llevaron a cabo en el Laboratorio de Suelos de la Universidad Nacional Agraria La Molina, en Setiembre del 2008. Las concentraciones por debajo del nivel de detección (con valor cero) fueron tomadas en cuenta para el análisis estadístico.

Para la comparación, se agrupó a los individuos por géneros, teniendo en cuenta que la estructura de gremios puede ser resultado de restricciones en el diseño estructural de las especies consumidoras, generalmente emparentadas (Pianka, 1980, citado por López, 2001). Esto se refleja en las similitudes de la dieta registradas en especies del mismo género (Muchhala \& Jarrin, 2002; Lou \& Yurrita, 2005; Gonçalves da Silva et al., 2008 y Mello et al., 2008). Para el presente estudio se consideró a Carollia como frugívoro-insectívoro (Cloutier \& Thomas, 1992), Anoura como omnívoro-insectívoro (Ortega \& Alarcón, 2008) y Sturnira como frugívoro (Giannini \& Barquez, 2003).

Para las comparaciones entre los tres géneros se utilizó la prueba de Kruskall-Wallis, y entre pares de géneros y épocas se utilizó la prueba de Mann Whitney, considerando un nivel de significancia $\alpha=0.05$. Además, se presentan promedios, desviaciones estándar y rangos totales de concentraciones de los metales pesados por especie. 
Para los análisis y la presentación estadística se utilizaron los programas PAST $^{\circledR}$ versión $1.71 \mathrm{y}$ MINITAB ${ }^{\circledR} 14$.

\section{Resultados y discusión.}

Diferencias entre los géneros.

Se determinó que para los metales $\mathrm{Cd}, \mathrm{Cu}$ y $\mathrm{Zn}$, por lo menos uno de los géneros presenta una concentración diferente (Tabla 1). Sin embargo, no se hallaron diferencias significativas en las concentraciones de $\mathrm{Pb}$ entre los tres géneros. Las concentraciones de $\mathrm{Cu}$ y $\mathrm{Zn}$ fueron mayores en Anoura, seguido por Sturnira y finalmente Carollia. El género Anoura presentó los valores más bajos de $\mathrm{Pb}$ y $\mathrm{Cd}$, mientras que Sturnira presentó el valor más alto de $\mathrm{Cd}$ y Carollia de $\mathrm{Pb}$ (Tabla 1).

En las comparaciones de pares de géneros realizadas con la prueba Mann-Whitney se evidencia similitud estadística $(\alpha=0.05)$ entre las concentraciones de $\mathrm{Cd}$ y $\mathrm{Cu}$ en los géneros Carollia y Sturnira (Anexo 1).

Tabla 1. Concentraciones de metales pesados (ppm) en los géneros más abundantes (medianas y rangos intercuartiles entre paréntesis). El p-value es la probabilidad que todas las medianas de los géneros sean similares (prueba KruskalWallis).

\begin{tabular}{ccccc}
\hline & Carollia $(14)$ & Anoura $(8)$ & Sturnira $(27)$ & $p_{K W}$ \\
\hline $\mathrm{Pb}$ & 1.53 & 0 & 0.77 & 0.836 \\
& $(0-3.15)$ & $(0-7.57)$ & $(0-4.1)$ & \\
$\mathrm{Cd}$ & 2.19 & 1 & 2.73 & 0.003 \\
& $(1.46-3.19)$ & $(0-1.52)$ & $(2.05-4.02)$ & \\
$\mathrm{Cu}$ & 19.07 & 50.64 & 24.24 & 0.001 \\
& $(16.88-26.59)$ & $(37.97-77)$ & $(18.58-37.1)$ & \\
$\mathrm{Zn}$ & 86.5 & 148 & 102.03 & 0.001 \\
& $(81.5-101.7)$ & $(122.7-241.5)$ & $(93.61-142.62)$ & \\
\hline
\end{tabular}

Diferencias entre ambas épocas

No se detectaron diferencias significativas para los géneros Carollia ni Sturnira entre ambas épocas. Debido al restringido número de individuos de Anoura, este género no fue tomado en cuenta para el análisis. Para la época lluviosa, ambos géneros presentaron mayores valores de $\mathrm{Pb}$ y menores de $\mathrm{Cd}$ y $\mathrm{Cu}$ (Tabla 2). El Zn mostró una tendencia diferente, siendo su concentración mayor en la época lluviosa para Carollia y menor para Sturnira (Tabla 2).

En ambos géneros se capturó un mayor número de individuos jóvenes durante la época lluviosa. En el género Carollia, el $56 \%$ de los individuos colectados fueron juveniles, mientras que para Sturnira, fue el 29 $\%$. En comparación, en la época seca, no se colectó ningún individuo joven perteneciente al género

\section{juveniles.}

A nivel de especies.

En total, se realizaron las lecturas de las concentraciones en 14 especies, de las cuales 11 pertenecen a los géneros Carollia, Anoura y Sturnira (Tabla 3). Las especies más abundantes fueron Sturnira oporaphyllum y Carollia brevicauda, con el $33 \%$ y $22 \%$ de individuos del total analizado, respectivamente.

En todas las especies, las concentraciones de $\mathrm{Zn}$ son mayores a las de $\mathrm{Cu}$, siendo ambas muy superiores a las de $\mathrm{Pb}$ y Cd (Tabla 3). En la totalidad de las muestras analizadas (54 individuos), se encontró $\mathrm{Pb}$ en un rango de $0 \mathrm{a}$ 15.15 ppm, Cd de 0 a 19.05 ppm, $\mathrm{Cu}$ de 4.38 a 81.82 ppm y $\mathrm{Zn}$ de 9.49 a 284 ppm. Un individuo de Anoura caudifer presentó valores elevados de Cd (12 ppm), y sumamente elevados de $\mathrm{Pb}(344 \mathrm{ppm})$ con respecto a los demás individuos.

Discusión.

Diferencias entre los géneros.

Tanto al $\mathrm{Pb}$ como al $\mathrm{Cd}$ no se les atribuye función fisiológica, siendo considerados como contribuyentes a la toxicidad cuando se eleva su ingesta (McDowell, 1992). Por ello, si bien ambos están presentes en la naturaleza y son parte de la historia natural de los ecosistemas, actúan como contaminantes al liberarse a la atmósfera como producto de las operaciones industriales (McDowell, 1992; Albert, 1997; Capó, 2002), y pueden ser asimilados por vía respiratoria en cantidades considerables. Como consecuencia, las concentraciones de $\mathrm{Pb}$ y $\mathrm{Cd}$ halladas en los murciélagos, dependerán, además de la dieta, de su grado de exposición al metal y de la exposición de las plantas e insectos consumidos por éstos.

Los resultados obtenidos sugieren que estos géneros presentarían similares niveles de exposición al 
$\mathrm{Pb}$, por lo que estarían siendo afectados con la misma intensidad. Lo contrario ocurre con las concentraciones de $\mathrm{Cd}$, que resultaron similares en Carollia y Sturnira, mientras que en Anoura fueron menores. La menor asimilación de $\mathrm{Cd}$ en este género, estaría asociada a sus hábitos alimentarios y a las diferencias fisiológicas de asimilación (Walker et al., 2007).

Tabla 3. Concentraciones totales (ppm) de metales pesados en hígado y páncreas de quirópteros colectados en el Lodge "Cock of the Rocks" y alrededores. (Media \pm desviación estándar y rango total entre paréntesis).

\begin{tabular}{lcccc}
\hline Especie (n) & $\mathrm{Pb}$ & $\mathrm{Cd}$ & $\mathrm{Cu}$ & $\mathrm{Zn}$ \\
\hline C. brevicauda (12) & $2.39 \pm 2.28$ & $2.52 \pm 1.18$ & $22.22 \pm 9.66$ & $88.48 \pm 38.9$ \\
& $(0-6.62)$ & $(1.28-7.9)$ & $(4.38-40.52)$ & $(9.49-181.58)$ \\
C. manu (1) & 0 & 7.89 & 23.68 & 147.37 \\
C. perspicillata (1) & 0 & 2.35 & 15.29 & 85.88 \\
A. cf. caudifer (1) & 0 & 0 & 41.27 & 120.64 \\
A. geoffroyi (2) & $0 ; 9.09$ & $0 ; 2.02$ & $37.5 ; 39.39$ & $166.67 ; 259.38$ \\
A. cultrata (1) & 0 & 0 & 19.51 & 129.27 \\
A. caudifer (4) & $86.75 \pm 171.51$ & $3 \pm 6$ & $73.45 \pm 9.52$ & $176.39 \pm 79.84$ \\
& $(0-344)$ & $(0-12)$ & $(60-81.82)$ & $(104.54-284)$ \\
S. erythromos (5) & $4.65 \pm 6.12$ & $2.73 \pm 1.12$ & $31.47 \pm 9.47$ & $114.84 \pm 44.37$ \\
& $(0.54-15.15)$ & $(1.61-4.55)$ & $(18.58-40)$ & $(42.47-163.64)$ \\
S. lilium (3) & $2.41 \pm 2.33$ & $7.06 \pm 6.41$ & $32.26 \pm 10.03$ & $150.79 \pm 28.41$ \\
& $(0-4.65)$ & $(3.23-14.47)$ & $(20.93-40)$ & $(118.02-168.81)$ \\
S. oporaphyllum (18) & $2.21 \pm 3.66$ & $3.9 \pm 4.22$ & $27.5 \pm 13.7$ & $135.44 \pm 32.18$ \\
& $(0-14.29)$ & $(1.06-19.05)$ & $(15.74-69.67)$ & $(74.75-188.78)$ \\
S. tildae (1) & 0.73 & 4.02 & 26.64 & 94.89 \\
P. stenops (1) & 0.59 & 0.79 & 26.72 & 78.19 \\
A. cinereus (1) & 3.77 & 0 & 39.62 & 113.21 \\
P. masu (3) & $0.48 \pm 0.83$ & $1.06 \pm 1.1$ & $17.28 \pm 1.74$ & $80.47 \pm 10.22$ \\
& $(0-1.44)$ & $(0-2.19)$ & $(15.83-19.21)$ & $(69.97-88.49)$ \\
\hline
\end{tabular}

A diferencia de los elementos anteriores, el $\mathrm{Cu}$ y el $\mathrm{Zn}$ cumplen determinadas funciones fisiológicas (Eckert \& Randall, 1990). Estos nutrientes deben ser absorbidos y eliminados constantemente, manteniéndose niveles de homeostasis ya que en exceso pueden ser tóxicos y al ser escasos se pueden presentar deficiencias (McDowell, 1992; Prohaska \& Gybina, 2004). Su absorción se da principalmente por vía digestiva, lo que está relacionado directamente al tipo de dieta.

Las concentraciones de $\mathrm{Cu}$ fueron similares en Carollia y Sturnira, siendo menores a las encontradas en Anoura. Posiblemente, esto se deba al mayor consumo de insectos por parte de Anoura respecto a los otros dos géneros que son más frugívoros. Según Hoenerhoff \& Williams (2004), generalmente las frutas tienen bajos niveles de $\mathrm{Cu}$. Además, los murciélagos acumulan este metal después de alimentarse de insectos que han terminado la fase acuática de su ciclo vital en agua contaminada. Dada la similitud estadística entre Carollia y Sturnira, consideradas como especialistas en Piperáceas y Solanáceas respectivamente (Lou \& Yurrita, 2005; Mello et al., 2008), se tiene que Sturnira presenta mayores concentraciones. Aunque esto no representa suficiente evidencia, es posible que las Solanáceas hayan proporcionado mayor contenido de $\mathrm{Cu}$ que las Piperáceas, aún cuando Carollia complementa su dieta con insectos más frecuentemente que Sturnira.

Los mamíferos presentan una limitada capacidad para almacenar $\mathrm{Zn}$, por lo que la concentración interna de este metal se encuentra generalmente relacionada a su contenido en la dieta (McDowell, 1992; Milton \& Johnson, 1999). Los resultados reflejan esto último, ya que las concentraciones de $\mathrm{Zn}$ son diferentes en los tres géneros, siendo mayores en Anoura, seguidas por Sturnira y finalmente por Carollia. Aparentemente, las concentraciones de $\mathrm{Zn}$ seguirían una jerarquía similar a la que ocurre con el $\mathrm{Cu}$, siendo mayor en insectos, seguida por Solanáceas y finalmente Piperáceas.

Diferencias entre ambas épocas.

Para Carollia y Sturnira, se registran mayores concentraciones $\mathrm{de} \mathrm{Pb}$ en la época lluviosa, en la que se presentan temperaturas más elevadas, lo que provoca una disminución en su excreción por parte de los individuos (Capó, 2002). Para la misma época, se dan menores concentraciones de $\mathrm{Cd}$. Como este metal se asimila por la vía respiratoria, la menor concentración hallada podría estar relacionada con cambios en los vientos o con el grado de emanación de contaminantes (Albert, 1997). Por otro lado, el Cd se bioacumula con la edad (McDowell, 1992; Shore \& Rattner, 2001, citados por Walker et al., 2007), por lo que las bajas concentraciones halladas en la época lluviosa, podrían ser reflejo del mayor número de juveniles capturados en dicha época. Sin embargo, al hacer el análisis por sexos (Anexo 2), no se encontraron menores concentraciones en los machos, donde había una mayor proporción de juveniles. Por ello, se sugiere que se estaría dando una menor asimilación de $\mathrm{Cd}$ por parte de las hembras que podría relacionarse con algún factor de su comportamiento (Thies et al., 2006).

Las menores concentraciones de $\mathrm{Cu}$ para ambos géneros en la época lluviosa, puede deberse a la reducción de la captación de dicho metal por parte de las plantas ante las condiciones reductoras imperantes (Espinoza, 2000). Por lo tanto, si los frutos tienen 
menores concentraciones de $\mathrm{Cu}$, es de esperarse una menor asimilación de $\mathrm{Cu}$ en los animales.

Dado que en los mamíferos el principal factor que afecta la absorción del $\mathrm{Zn}$ es el contenido en la dieta (McDowell, 1992), las mayores concentraciones de $\mathrm{Zn}$ halladas en el género Carollia en la época lluviosa podrían deberse a sus hábitos alimenticios (Gardner, 1977) y a la interacción suelo - planta. Se sabe que el $\mathrm{Zn}$ aumenta su solubilidad en condiciones reductoras, hecho que facilitaría su captación por parte de las plantas en la época lluviosa (Loué, 1988, citado por Espinoza, 2000). Sin embargo, lo que ocurre en Sturnira es lo contrario, hallándose menores valores de $\mathrm{Zn}$ para dicha época. Estas diferencias podrían trazarse nuevamente a los hábitos alimenticios. Sturnira estaría modificando sus hábitos alimentarios consumiendo frutos complementarios de la familia de las Piperáceas y por lo tanto presentando menores concentraciones de Zn (Mello et al., 2008; Mikich et al., 2003).

A nivel de especies.

Aparentemente estos datos de base serían los primeros reportes sobre concentraciones de $\mathrm{Pb}, \mathrm{Cd}$, $\mathrm{Cu}$ y $\mathrm{Zn}$ en murciélagos de Sudamérica. Los rangos aquí presentados son similares a los mencionados en Hoenerhoff \& Williams (2004) y Walker et al. (2007), para $\mathrm{Cu}$ hepático y $\mathrm{Pb}$ y $\mathrm{Cd}$ renales. Además, las mayores concentraciones de $\mathrm{Cu}$ y $\mathrm{Zn}$ y menores de $\mathrm{Pb}$ y $\mathrm{Cd}$ concuerdan con los trabajos realizados en pequeños mamíferos por Milton \& Johnson (1999) y Lodenius et al. (2002). Esto se relaciona con el hecho que en general, los mamíferos requieren mayores cantidades de $\mathrm{Cu}$ y $\mathrm{Zn}$ para suplir sus necesidades fisiológicas.

En este estudio se encontró que un individuo de Anoura caudifer presentó altos niveles de $\mathrm{Pb}$ y $\mathrm{Cd}$. Hoenerhoff \& Williams (2004) en un trabajo similar, atribuyeron altos valores de un metal a las características individuales. En este caso, el individuo pudo estar expuesto a condiciones extremas y por tanto aumentó su ingesta, o tuvo dificultades para la excreción.

Las diferencias en las concentraciones de metales entre especies pueden ocurrir debido al grado de exposición, tipo de dieta y variaciones de asimilación (Hoenerhoff \& Williams, 2004; Walker et al., 2007), factores que no se tuvieron en cuenta en este estudio y que escapan a su alcance. Para un mayor entendimiento de las concentraciones óptimas, deficientes o tóxicas de metales pesados en estos mamíferos, sería necesario realizar estudios completos cuantitativos de sus hábitos alimenticios. Además, sería necesario realizar estudios exhaustivos de la composición química del suelo, las concentraciones de metales en las frutas e insectos consumidos por estos murciélagos, realizar estos análisis en otros tejidos y hacer comparaciones con individuos de áreas controles y otras muy contaminadas.

\section{Conclusiones.}

Los resultados aquí presentados serían los primeros sobre concentraciones de metales pesados en el hígado y páncreas de murciélagos en Sudamérica y servirán para posteriores estudios ecológicos y comparativos.

Los menores valores de $\mathrm{Pb}$ y $\mathrm{Cd}$ indicarían que estos metales serían absorbidos en gran medida por la vía respiratoria, mientras que los mayores valores de $\mathrm{Cu}$ y $\mathrm{Zn}$ señalarían que son obtenidos por ingestión, cumpliendo ciertas funciones fisiológicas.

Entre las especies, la absorción o excreción de estos metales serían afectadas tanto por el grado de exposición como por las características individuales de los animales.

Las diferencias en las concentraciones de los metales entre los géneros de murciélagos analizados estarían asociadas al tipo de dieta.

Las variaciones entre las épocas, halladas solamente para algunos géneros, podrían atribuirse a la disponibilidad de alimentos, la entrada de minerales al entorno y a la distinta absorción de metales por parte de las plantas.

Los resultados obtenidos en este trabajo, estarían señalando el camino de futuras investigaciones en este tema.

\section{Agradecimientos.}

Al Blgo. Daniel Blanco (Perú Verde), al Ph.D. David Goldstein (UPCH), al laboratorio de Estudios en Biodiversidad (UPCH), al laboratorio de Fisiología Animal y Biorremediación Universidad Nacional Agraria La Molina (UNALM), y al laboratorio de Suelos (UNALM) por el financiamiento otorgado durante las distintas etapas de este proyecto. A la Blga. Jessica Amanzo (UPCH), Aaron Canepa, Blga. Carla Delgado, Jorge León, Rossana Maguiña, Alessandra Quiñónez y Blga. Cindy Vergel, por su confianza, enseñanzas y las facilidades en las salidas de campo y en el trabajo de gabinete. Así mismo a todos aquellos profesionales y especialistas que aportaron al desarrollo de este trabajo, especialmente a los profesores Ever Menacho (UNALM) y Ana Vargas (UNALM). A la Biblioteca Agrícola Nacional (BAN-UNALM) por su apoyo y al personal del Lodge "Cock of the Rocks" por su hospitalidad.

\section{Literatura citada.}

Albert L. 1997. Curso básico de toxicología ambiental. Editorial Limusa, México.

Ahumada R. 1994. Nivel de concentración e índice de bioacumulación para metales pesados $(\mathrm{Cd}, \mathrm{Cr}, \mathrm{Hg}, \mathrm{Ni}$, $\mathrm{Cu}, \mathrm{Pb}$ y $\mathrm{Zn}$ ) en tejidos de invertebrados bénticos de Bahía San Vicente, Chile. Revista de Biología Marina. 29(1): 77-87.

Capó M. 2002. Principios de Ecotoxicología: diagnóstico, tratamiento y gestión del medio ambiente. Mc Graw Hill. Madrid. 
Cloutier D. \& Thomas D. 1992. Carollia perspicillata. Mammalian Species 417: 1-9.

Delgado C. 2010. Variación estacional en la diversidad y estado reproductivo de quirópteros con énfasis en los géneros Sturnira y Carollia (Chiroptera: Phyllostomidae) en el bosque nublado de San PedroCusco. Tesis para optar el grado de Bióloga. UPCH.

Eckert R. \& Randall D. 1990. Fisiología animal, mecanismos y adaptaciones. Interamericana Mc GrawHill. Madrid.

Espinoza G. 2000. Tolerancia a la toxicidad de zinc, cobre y plomo en plantas silvestres asociadas a suelos contaminados por la actividad minera. Tesis para optar el grado de Biólogo. Universidad Nacional Agraria La Molina Lima, Perú.

Gardner A. 1977. Feeding Habits. Pp: 293-350. En R.J. Baker, J.K. Jones, Jr., \& D. C. Carter (Eds.) Biology of bats of the new world, family Phyllostomidae. Part II, Spec. Public. Mus. Texas Tech Univ., 13.

Giannini N. \& Barquez R. 2003. Sturnira erythromos. American Society of Mammalogists. 729: 1-5.

Gil M., Torres A., Harvey M. \& Esteves J. 2006. Metales pesados en organismos marinos de la zona costera de la Patagonia argentina continental. Revista de Biología Marina y Oceanografía. 41(2): 167-176.

Gonçalves da Silva A., Gaona O. \& Medellín R. 2008. Diet and trophic structure in a community of fruit-eating bats in Lacandon forest, México. Journal of Mammalogy. 89(1): 43-49.

Hoenerhoff M. \& Williams K. 2004. Copper-associated hepatopathy in a Mexican fruit bat (Artibeus jamaicensis) and establishment of a reference range for hepatic copper in bats. J Vet Diagn Invest. 16: 590-593.

INRENA \& Pro Manu. 2002. Plan Maestro del Parque Nacional del Manu. Perú. Equipo técnico: Luis Palma, Gustavo Ruiz y Wilfredo Chávez.

Jacinto M. \& Aguilar S. 2007. Concentraciones traza de metales en especies marinas de la bahía de Huarmey, Ancash, Perú. Revista Peruana de Biología. 14(2): 307311.

Lodenius M., Soltanpour-Gargari A., Tulisalo E. \& Henttonen H. 2002. Effects of ash application on cadmium concentration in small mammals. J. Environ Qual. 31: 188- 192.

López J. 2001. Estructura gremial y organización de un ensamble de aves del desierto del monte. Tesis para optar el titulo de doctor en ciencias biológicas. UBA.
Lou S. \& Yurrita C. 2005. Análisis de nicho alimentario en la comunidad de murciélagos frugívoros de Yaxhá, Petén, Guatemala. Acta Zoológica Mexicana. 21(1): 8394.

McDowell L. 1992. Minerals in Animal and Human Nutrition. Academic Press, Inc. Harcourt Brace Jovanocich Publishers, California.

Mello M., Kalko E. \& Silva W. 2008. Diet and abundance of the bat Sturnira lilium (Chiroptera) in a Brazilian montane Atlantic forest. Journal of Mammalogy. 89(2): 485-492.

Mikich S., Bianconi G., Sales Maia, B. \& Teixeira, S. 2003. Attraction of the Fruit-Eating Bat Carollia perspicillata to Piper gaudichaudianum Essential Oil. Journal of Chemical Ecology. 41 (2): 2379-2383.

Milton A. \& Johnson M. 1999. Biomonitoring of contaminated mine tailings through age accumulation of trace metals in the bank vole (Clethrionomys glareolus). J. Environ. Monit. 1: 219-225.

Muchhala N. \& Jarrín-V P. 2002. Flower visitation by bats in could forests of Western Ecuador. Biotropica. 34(3): 387-395.

Municipalidad Provincial de Paucartambo. 2006. Plan de desarrollo concertado de la provincia de Paucartambo 2006-2010. Consultor: José Vásquez; comité técnico: Dionel Vizcardo, Rosa Álvarez, Araceli Roldan, Juan la Torre, Edson Jiménez y Anibal Pacheco.

Ortega J. \& Alarcón-D I. 2008. Anoura geoffroyi (Chiroptera: Phyllostomidae). Mammalian Species. 818: $1-7$.

Patterson B., Pacheco V. \& Solari S. 1996. Distributions of bat along an elevational gradient in the Andes of southeastern Perú. The Zoological Society of London. 240: 637-658

Prohaska J. \& Gybina A. 2004. Intracellular copper transport in mammals. American Society Nutritional sciences. J. Nutr. 134: 1003-1006.

Thies W., Kalko E. \& Schnttzler H. 2006. Influence of environment and resource availability on activity patterns of Carollia castanea (Phillostomidae) in Panama. Journal of Mammalogy. 87 (2): 331-338.

Walker L., Simpson V., Rockett L., Wienburg C. \& Shore R. 2007. Heavy metal contamination in bats in Britain. Environmental Pollution. 148: 483-490. 
Anexo 1. Comparaciones entre pares de géneros. El pvalue es la probabilidad que las medianas de los pares de géneros sean similares (prueba Mann-Whitney).

\begin{tabular}{lccc}
\hline & $\boldsymbol{p}_{\boldsymbol{C d}}$ & $\boldsymbol{p}_{\boldsymbol{C u}}$ & $\boldsymbol{p}_{\text {Zn }}$ \\
\hline Carollia VS Anoura & 0.013 & 0.0012 & 0.0015 \\
Carollia VS Sturnira & 0.0857 & 0.1048 & 0.0269 \\
Anoura VS Sturnira & 0.0023 & 0.003 & 0.0095 \\
\hline
\end{tabular}

Anexo 2. Concentraciones de metales pesados (ppm) según el sexo en los tres géneros más abundantes (medianas y rangos intercuartiles).

\begin{tabular}{|c|c|c|c|c|}
\hline Género & $\mathrm{Pb}$ & $\mathrm{Cd}$ & $\mathrm{Cu}$ & $\mathrm{Zn}$ \\
\hline & \multicolumn{4}{|c|}{ Hembras } \\
\hline Carollia (5) & $\begin{array}{c}1.75 \\
(0.65-5.27)\end{array}$ & $\begin{array}{c}1.52 \\
(1.37-5.58)\end{array}$ & $\begin{array}{c}18.94 \\
(11.16-38.68)\end{array}$ & $\begin{array}{c}84.6 \\
(32.5-142.3)\end{array}$ \\
\hline Anoura (2) & $0 ; 3$ & $0 ; 0$ & $41.27 ; 74$ & $120.64 ; 129$ \\
\hline \multirow[t]{2}{*}{ Sturnira (18) } & $\begin{array}{c}0.7 \\
(0-4.22)\end{array}$ & $\begin{array}{c}2.69 \\
(2.05-3.64)\end{array}$ & $\begin{array}{c}26.57 \\
(17.66-38.08)\end{array}$ & $\begin{array}{c}109.26 \\
(91.76-160.33)\end{array}$ \\
\hline & \multicolumn{4}{|c|}{ Machos } \\
\hline Carollia (9) & $\begin{array}{c}0.79 \\
(0-2.62)\end{array}$ & $\begin{array}{c}2.35 \\
(1.74-2.77)\end{array}$ & $\begin{array}{c}19.19 \\
(16.77-24.94)\end{array}$ & $\begin{array}{c}87.16 \\
(83.85-99.8)\end{array}$ \\
\hline Anoura (6) & $\begin{array}{c}0 \\
(0-92.8)\end{array}$ & $\begin{array}{c}0 \\
(0-4.52)\end{array}$ & $\begin{array}{c}49.7 \\
(33-79)\end{array}$ & $\begin{array}{c}177.3 \\
(123.1-265.5)\end{array}$ \\
\hline Sturnira (9) & $\begin{array}{c}1.08 \\
(0.53-8.06)\end{array}$ & $\begin{array}{c}3.05 \\
(2.27-4.28)\end{array}$ & $\begin{array}{c}24.24 \\
(20.10-35.61)\end{array}$ & $\begin{array}{c}102 \\
(95.3-127.3)\end{array}$ \\
\hline
\end{tabular}

\footnotetext{
${ }^{1}$ Departamento Académico de Biología. Facultad de Ciencias. Universidad Nacional Agraria la Molina, mwilliams@lamolina.edu.pe

${ }^{2}$ Universidad Nacional Agraria la Molina. cocha_huayco@hotmail.com

${ }^{3}$ Universidad Nacional Agraria la Molina.

${ }^{4}$ Departamento Académico de Suelos. Facultad de Agronomía. Universidad Nacional Agraria la Molina. braulio@lamolina.edu.pe
} 